\title{
Asteroseismology of OB stars with CoRoT*
}

\author{
P. Degroote ${ }^{1, \star \star}$, C. Aerts $^{1,2}$, R. Samadi ${ }^{3}$, A. Miglio $^{4}$, M. Briquet ${ }^{1 \circ}$, M. Auvergne $^{3}$, A. Baglin ${ }^{5}$, \\ F. Baudin ${ }^{6}$, C. Catala ${ }^{3}$, and E. Michel ${ }^{7}$ \\ 1 Instituut voor Sterrenkunde, K.U.Leuven, Celestijnenlaan 200D, B-3001 Leuven, Belgium \\ 2 Department of Astrophysics, IMAPP, University of Nijmegen, PO Box 9010, 6500 GL Nijmegen, The Netherlands \\ 3 LESIA, UMR8109, Université Pierre et Marie Curie, Université Denis Diderot, Observatoire de Paris, 92195 Meudon \\ Cedex, France \\ ${ }^{4}$ Institut d'Astrophysique et de Géophysique Université de Liège, Allée du 6 Août 17, B 4000 Liège, Belgium \\ ${ }^{5}$ Laboratoire AIM, CEA/DSM-CNRS-Université Paris Diderot; CEA, IRFU, SAp, centre de Saclay, F-91191, Gif-sur- \\ Yvette, France \\ ${ }^{6}$ Institut d'Astrophysique Spatiale (IAS), Bâtiment 121, F-91405, Orsay Cedex, France \\ 7 LESIA, CNRS, Université Pierre et Marie Curie, Université Denis Diderot, Observatoire de Paris, 92195 Meudon cedex, \\ France
}

Received 2010 Sep 9, accepted 2010 Sep 13

Published online 2010 Nov 11

Key words methods: data analysis - stars: individual (HD 46179, HD 50230, HD 180642) - stars: oscillations - stars: interiors - techniques: photometric

The CoRoT satellite is revolutionizing the photometric study of massive O-type and B-type stars. During its long runs, CoRoT observed the entire main sequence B star domain, from typical hot $\beta$ Cep stars, via cooler hybrid $\mathrm{p}$ - and g-mode pulsators to the SPB stars near the edge of the instability strip. CoRoT lowers the sensitivity barrier from the typical mmagprecision reached from the ground, to the $\mu$ mag-level reached from space. Within the wealth of detected and identified pulsation modes, relations have been found in the form of multiplets, combination of frequencies, and frequency- and period spacings. This wealth of observational evidence is finally providing strong constraints to test current models of the internal structure and pulsations of hot stars. Aside from the expected opacity driven modes with infinite lifetime, other unexpected types of variability are detected in massive stars, such as modes of stochastic nature. The simultaneous observation of all these light curve characteristics implies a challenge for both observational asteroseismology and stellar modelling.

\section{Introduction}

Stars with masses above a few solar masses are cornerstones in the field of stellar and galactic evolution. They evolve orders of magnitude faster than lighter stars like our own sun, and are the main factories of elements heavier than those produced by cosmological nucleosynthesis. Exactly how much of these processed elements are fed back to the interstellar medium, is highly dependent on their initial and final mass, and therefore also on their evolution and internal structure.

A key observation for unraveling the internal structure of massive stars is the fact that at least some of them pulsate. Although we cannot invert the observed pulsations to obtain a model for the stellar interior, as is possible for the sun (Christensen-Dalsgaard et al. 1991), we can, in principle, confront observed pulsations with their theoretical counter

\footnotetext{
* The CoRoT space mission was developed and is operated by the French space agency CNES, with participation of ESA's RSSD and Science Programmes, Austria, Belgium, Brazil, Germany, and Spain.

$\star \star$ Corresponding author: pieterd@ster.kuleuven.ac.be

- Postdoctoral Fellow of the Fund for Scientific Research, Flanders
}

parts (e.g., Aerts et al. 2010). A mismatch between theory and observations then implies a change in the relevant input physics, leading to a refinement of current stellar models.

In the $\log T_{\text {eff }}-\log g$ (effective temperature vs. surface gravity) diagram (Fig. 1) populated by OB-type stars, two regions of pulsational instability are predicted and observed. In both instability strips, the pulsations are driven by the $\kappa$ mechanism (Dziembowski \& Pamiatnykh 1993), related to the metal opacity bump around $T \approx 2 \times 10^{5} \mathrm{~K}$, where loworder modes are excited. In the coolest of the two strips, low frequency gravity modes (roughly between 3 and $12 \mu \mathrm{Hz}$ ) are observed (so called SPB stars), while in the hotter instability strip, pressure modes with frequencies in the range of $30-90 \mu \mathrm{Hz}$ are found to be common ( $\beta$ Cep stars). The extent of the instability strips towards hotter stars, is still largely unknown.

For completeness, we note that pulsations are not the only type of variability in massive stars, nor are they the sole source of classification; many other features co-exist, such as pulsating and non-pulsating emission line $(\mathrm{Oe} / \mathrm{Be})$ stars and chemically peculiar (Op/Bp) stars (e.g., Walborn \& Fitzpatrick 2000). 


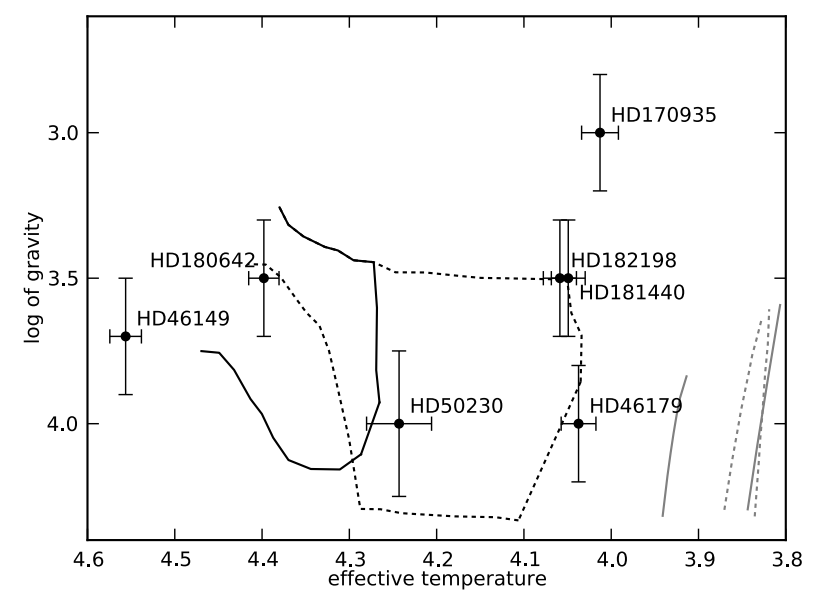

Fig. 1 Observational $\log T_{\text {eff }}-\log g$ diagram with the two predicted instability strips (SPB, dashed line, $\beta$ Cep, solid line) and a selection of CoRoT targets, using OPAL opacities and a metallicity of $Z=0.02$. For reference, also the $\gamma$ Dor $/ \delta$ Sct instability strips are shown (grey dashed/solid lines).

Current knowledge states that OB-type stars consist of a radiative envelope around a convective core, although convection could in principle also exist in small regions where the opacity attains a local maximum, and the oscillations are driven by turbulent convection (Belkacem et al. 2010). However, many uncertainties still exist in the physical description of the processes occurring inside the star, e.g. concerning the size of the convective core, the value of the overshoot parameter needed in describing convection, the nature of the mixing processes around the core, the distribution of the internal angular momentum and mode selection mechanisms. B-type stars are invaluable, because the existence of gravity and pressure modes allows us, at least in principle, to probe the structure of the star from the core to the surface.

To this end, continuous and high duty-cycle observations of OB-type stars are part of the asteroseismology programme (Michel et al. 2006) of the CoRoT space satellite (Baglin et al. 2002). In the following sections, we explore and review a subsample (Fig. 1) of these observations. Sections 1.1 and 1.2 are published in more detail as Degroote et al. (2010) and Degroote et al. (2009).

\subsection{The SPB star HD 50230}

HD 50230 is a B3 V main sequence star. Before the CoRoT observations, little was known about the target. Using spectra from the 1.2-m twin telescopes CORALIE (Baranne et al. 1996) and HERMES (Raskin \& Van Winckel 2008) on La Silla (Chile) and La Palma (Spain), combined with literature photometry, the fundamental parameters were derived to be $T_{\text {eff }}=17500 \pm 1500 \mathrm{~K}$ and $\log g=3.8 \pm 0.2$, putting the star near the common part of the SPB- $\beta$ Cep instability strips. The hybrid $\beta$ Cep-SPB nature of HD 50230 is also evident from the CoRoT light curve (Fig. 2), where few and low-amplitude $\mathrm{p}$ modes are detected alongside tens

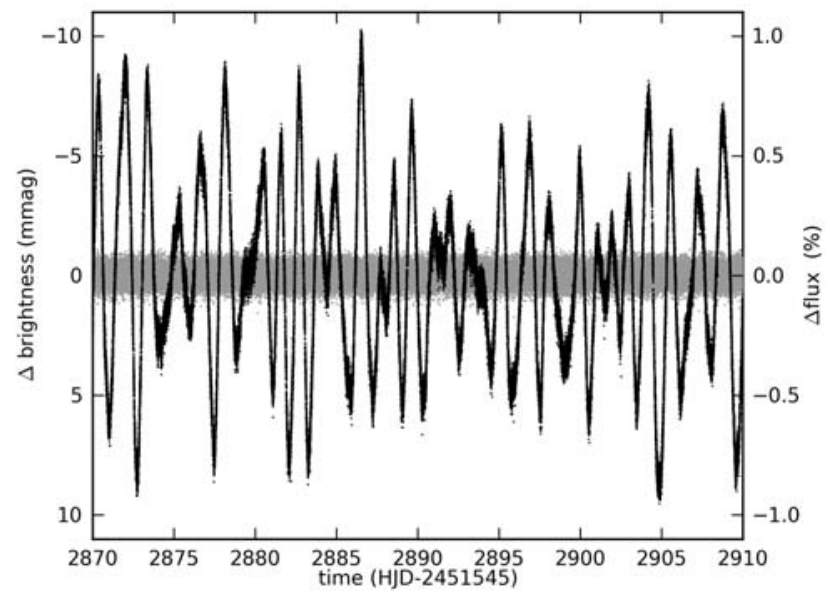

Fig. 2 Excerpt of the CoRoT light curve of HD 50230 (black dots). In grey, the residuals after subtracting all significant frequencies, are shown (adapted from Degroote et al. 2010).

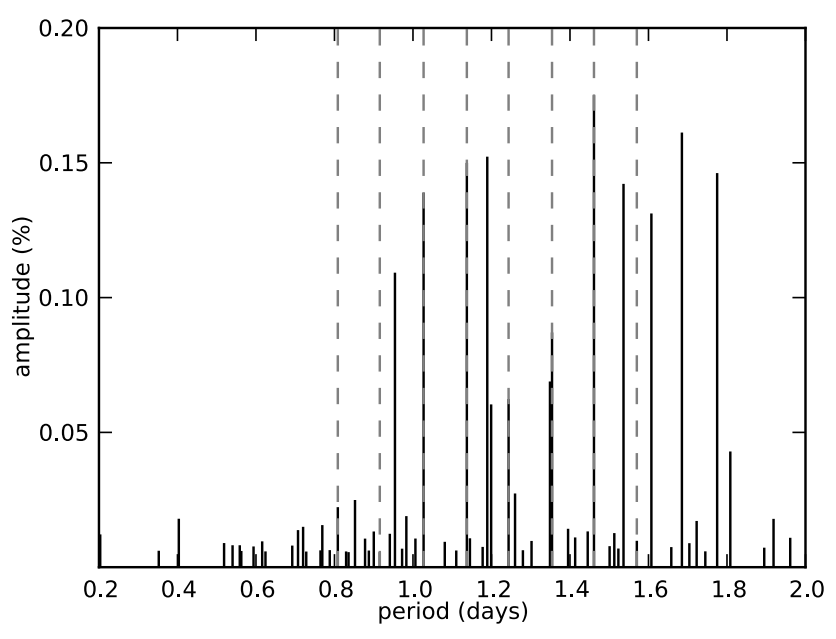

Fig. 3 Frequency spectrum of the g-mode region of HD 50230 (black solid lines). The vertical dashed lines represent the sequence of period spacings (adapted from Degroote et al. 2010).

to possibly hundreds of gravity modes, with amplitudes up to $1 \mathrm{mmag}$.

After extracting all frequencies using classical Fourier analysis techniques, some structure is noticeable in the forest of gravity mode peaks: a sequence of eight regularly spaced period peaks is identified (Fig. 3). A mean period spacing of $9418 \mathrm{~s}$ is found, where an oscillatory component is found to be present in the deviations.

To confront the observations with theory, appropriate models were computed with the Code Liégeois d'Évolution Stellaire (CLÉS, Scuflaire et al. 2008). Models were computed assuming solar metallicity, first without overshooting and turbulent mixing, but second also with an overshooting parameter between $\alpha=0.1-0.3^{1}$ or parametrized turbulent mixing.

\footnotetext{
1 The extension of the overshooting layer in the models is defined as $\alpha H_{\mathrm{p}}\left(r_{\mathrm{c}}\right)$, where $H_{\mathrm{p}}$ is the local pressure scaleheight at the border of the convective core $\left(r_{\mathrm{C}}\right)$
} 


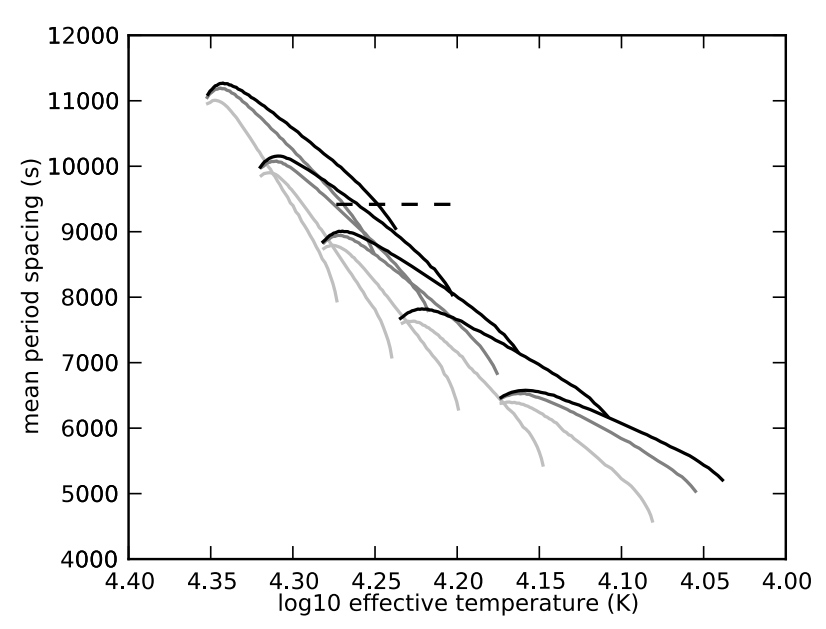

Fig. 4 The mean period spacing offers a constraint on the size of the convective core. The solid lines show the influence of the overshoot parameter on the mean period spacing for stellar models with masses between 4 and 8 solar masses $\left(\mathrm{M}_{\odot}\right)$ (no overshoot (light grey), $\alpha=0.2$ (dark grey), $\alpha=0.3$ (black). The dashed black line indicates the observed period spacing and an estimate of $T_{\text {eff }}$ (adapted from Degroote et al. 2010).

The period spacing and oscillatory component in the gravity modes allow us to derive several properties of the stellar core (Miglio et al. 2008). First, the mean period spacing, together with an estimate of the fundamental parameters, gives us an estimate of the overshoot parameter $\alpha \approx 0.3$ (Fig. 4). Moreover, assuming dipolar modes, the $200 \mathrm{~s}$ variations around the mean are interpreted as the signature of a chemically inhomogeneous region around the convective core, originating from the contraction of the core during its evolution on the main sequence.

\subsection{The $\beta$ Cep star HD 180642}

HD 180642 (V 1449 Aql) is a B1.5 II-III star, established as a member of the $\beta$ Cep class by Waelkens et al. (1998). Its dominant mode $\left(f_{1}=63.5056 \mu \mathrm{Hz}\right)$ having an amplitude of $\sim 0.08 \mathrm{mag}$, was identified by Aerts (2000) as radial. Following this discovery, an extensive ground based photometric and spectroscopic survey was set up (Briquet et al. 2009) to add frequencies, perform mode identification, and determine the element abundances in detail.

To increase the resolved frequencies of this large amplitude oscillator, the CoRoT space satellite has observed HD 180642 continuously for $\sim 130$ days. From the light curve, a broad range of frequencies is extracted, up to $\sim 230 \mu \mathrm{Hz}$. However, they are not independent; many of the high amplitude frequencies $f_{i}$ above $100 \mu \mathrm{Hz}$ can be written as a combination of two higher amplitude frequencies $f_{j}, f_{k} ; f_{i}=n_{j} f_{j}+n_{k} f_{k}+\epsilon$. In total 19 frequencies were unambiguously identified as combinations, where a deviation of the exact combination was allowed of (Fig. 5) $|\epsilon|<0.01 \mu \mathrm{Hz}$. These modes were interpreted as resonantly coupled to the high amplitude frequencies, of which $f_{1}$ is

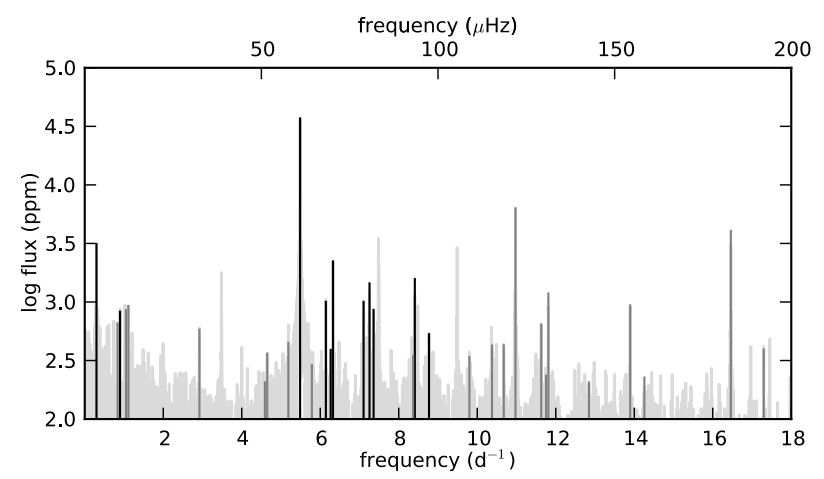

Fig. 5 Independent (black vertical bars) and dependent (grey vertical bars) frequencies in the spectrum of HD 180642. The raw frequency spectrum of the CoRoT light curve of HD 180642 is shown in grey in the background (adapted from Degroote et al. 2009).

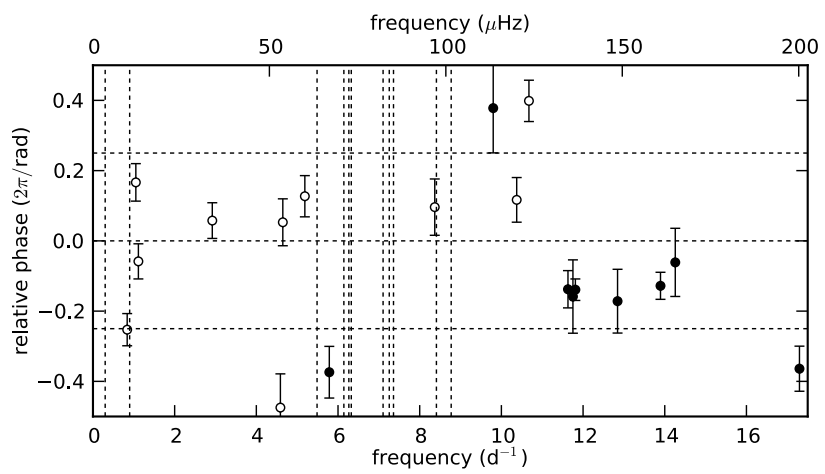

Fig. 6 Relative phases of dependent frequencies in HD 180642 as a function of frequency. The independent frequencies are indicated as vertical dashed lines. (filled symbols denote the frequencies with positive $n_{j}$ and $n_{k}$, open circles are show the frequencies for which one of $n_{j}$ or $n_{k}$ is negative (adapted from Degroote et al. 2009).

the most important one, with 15 coupled modes. This is argued by the observation that the dominant mode is nonlinear, which is already visible in the phase diagram. The fact that they are coupled is clearly seen from their common relative phase $\phi_{r}$ of frequency $f_{i}$, which is connected to the phases of frequencies $f_{j}$ and $f_{k}$ through (Fig. 6) $\phi_{r}=\phi_{i}-\left[n_{j} \phi_{j}+n_{k} \phi_{k}\right]$.

Besides the unstable modes, which are driven by the opacity mechanism, stochastically excited, solar-like oscillations were found in HD 180642 (Belkacem et al. 2009), adding asteroseismological constraints to stellar models of the star.

Given the wealth of frequencies and their relations, the fact that some of them are spectroscopically or photometrically determined, and the good determination of its spectroscopic parameters ( $T_{\text {eff }}, \log g$, projected equatorial rotation velocity $v \sin i$, and element abundances), we conclude that HD 180642 is a suitable target for detailed asteroseismological modelling, yet to be done.

From a first matching between model frequencies and observations, it is clear that the dominant mode, despite its 


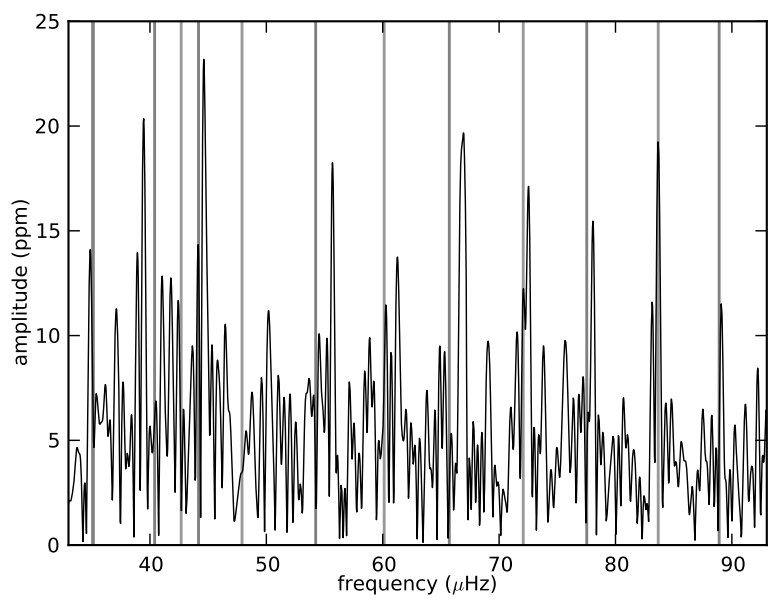

Fig. 7 Frequency spectrum of the CoRoT light curve of HD 46149 (black). The grey vertical lines denote the predicted frequencies for a model with a mass of $25.2 \mathrm{M}_{\odot}$, metallicity $Z=0.014$ and overshoot parameter $\alpha=0.15$.

large amplitude, cannot be the fundamental radial mode; instead a fairly high radial order $(n>3)$ is needed. Also, the large separation from models is not in agreement with the presumed highly evolved state of this $\beta$ Cep star.

\subsection{Beyond the classical instability strips}

The CoRoT satellite has also observed stars which fall on the border of the SPB- and $\beta$ Cep instability strips, or have evolved beyond it. It is thus no surprise that at least one star, HD 46179, does not seem to exhibit any type of variability, a side from (low amplitude) instrumental effects. In contrast, HD 181440 (spectral type B9 III), HD 182198 (B9 V), and HD 170935 (B8) are certainly variable, but here pulsations are either scarce or absent. Instead, these light curves are more comparable with those of spotted stars.

The O-star binary HD 46149, however, does show some pulsations, although less pronounced than HD 50230 and HD 180642. Here, clear signal below mmag level is present at low frequencies. This signature points towards rotational modulation, e.g., due to atmospheric activity, instead of pulsations. At higher frequencies, i.e. above $35 \mu \mathrm{Hz}$, a clear pattern of 11 regularly spaced frequencies is discovered. Moreover, there is clear evidence that these are not selfexcited but instead excited and damped (Fig. 7, Degroote et al., submitted). This finding effectively opens up this part of the HR-diagram for asteroseismology.

\subsection{Conclusion}

The presented selection of CoRoT targets shows the huge potential of high quality, high duty cycle space based observations of massive stars. In-depth study of specific targets has proven to be valuable, and general sample studies are starting to become possible, yielding a great potential to significantly expanding our knowledge of the structure and evolution of massive stars. For the time being, studies of $\mathrm{O}$ and hot B stars is a unique capability of CoRoT, as such pulsators have not yet been identified in the Kepler asteroseismology programme (Gilliland et al. 2010).

Acknowledgements. AM is a post-doctoral researcher of the Belgian FRS-FNRS. The results presented in this paper benefitted greatly from intensive discussions within the Belgian Asteroseismology Group (BAG, http://www.asteroseismology.be). The research leading to these results has received funding from the European Research Council under the European Community's Seventh Framework Programme (FP7/2007-2013)/ERC grant agreement $n^{\circ} 227224$ (PROSPERITY), from the Research Council of K.U. Leuven (GOA/2008/04), from the Fund for Scientific Research of Flanders (G.0332.06), and from the Belgian federal science policy office (C90309: CoRoT Data Exploitation).

\section{References}

Aerts, C.: 2000, A\&A 361, 245

Aerts, C., Christensen-Dalsgaard, J., Kurtz, D.W.: 2010, Asteroseismology, Springer

Baglin, A., Auvergne, M., Barge, P., et al.: 2002, in: B. Battrick, F. Favata, I.W. Roxburgh, D. Galadi (eds.), Stellar Structure and Habitable Planet Finding, ESA SP-485, p. 17

Baranne, A., Queloz, D., Mayor, M., et al.: 1996, A\&AS 119, 373

Belkacem, K., Samadi, R., Goupil, M.-J., et al.: 2009, Sci 324, 1540

Belkacem, K., Dupret, M.A., Noels, A.: 2010, A\&A 510, A6

Briquet, M., Uytterhoeven, K., Morel, T., et al.: 2009, A\&A 506, 269

Christensen-Dalsgaard, J., Gough, D.O., Thompson, M.J.: 1991, ApJ 378, 413

Degroote, P., Briquet, M., Catala, C., et al.: 2009, A\&A 506, 111

Degroote, P., Aerts, C., Baglin, A., et al.: 2010, Nature 464, 259

Dziembowski, W.A., Pamiatnykh, A.A.: 1993, MNRAS 262, 204

Gilliland, R.L., Brown, T.M., Christensen-Dalsgaard, J., et al.: 2010, PASP 122, 131

Michel, E., Baglin, A., Auvergne, M., et al.: 2006, in: M. Fridlund, A. Baglin, J. Lochard, L. Conroy (eds.), The CoRoT Mission Pre-Launch Status - Stellar Seismology and Planet Finding, ESA SP-1306, p. 39

Miglio, A., Montalbán, J., Noels, A., Eggenberger, P.: 2008, MNRAS 386, 1487

Raskin, G., Van Winckel, H.: 2008, in: I.S., McLean, M.M. Casali (eds.), Ground-based and Airborne Instrumentation for Astronomy II, SPIE 7014, p. 70145D

Scuflaire, R., Théado, S., Montalbán, J., et al.: 2008, Ap\&SS 316, 83

Waelkens, C., Aerts, C., Kestens, E., Grenon, M., Eyer, L.: 1998, A\&A 330, 215

Walborn, N.R., Fitzpatrick, E.L.: 2000, PASP 112, 50 